\title{
Do We Create Mathematics or Do We Gradually Discover Theories Which Exist Somewhere Independently of Us?
}

Vladimir L. Popov (Steklov Mathematical Institute, Moscow, Russia), Editor of EMS Newsletter

The headline question is a phrase from the article "Mathematics: Art and Science" by A. Borel, which is the English translation of the text of his lecture delivered (in German) in Munich in 1981. I remembered about this article in October 2016 when I saw the title "Discoveries, not inventions - Interview with Ernest B. Vinberg" of an article for publication in the EMS Newsletter, No. 102, December 2016 [2]. The very title clearly implies a definite answer to this question but it turns out that, in the interview, the issue of whether mathematicians explore something that exists independently of them or something they have invented is not discussed. However, as is shown in the article by A. Borel, this issue is, in fact, deeper than it may seem and contains items for discussion that might engage readers of the EMS Newsletter. Because of this, the mentioned article by A. Borel is reprinted below. There is also another reason to do this: in addition to a discussion of the formulated question, in this article, A. Borel also discusses other principal issues of a general nature related to mathematics, such as the problem of its relationship with the natural and applied sciences. The severity of the statements on these issues does not abate with time: to the citations in the reprinted paper by A. Borel, one could add the well known viewpoint of V. Arnold (and see also the recent interview with the Fields Medallist S. Novikov [3]). These are the items for discussion that might engage readers of the EMS Newsletter. Note that the reprinted article by A. Borel is not his only public statement on the subject (see [1]).

\section{References}

[1] A. Borel, On the place of mathematics in culture (Faculty lecture given at the Institute for Advanced Study, Oct. 16, 1991), Euvres, Vol. IV, 1983-1999, Springer, 419-440.

[2] A. Fialowski, J. Hilgert, B. Ørsted, V. Salnikov, Discoveries, not inventions - Interview with Ernest B. Vinberg, EMS Newsletter 102 (2016), 31-34.

[3] E. Kudryavtseva, The breakup of the mandatory knowledge had happen - Interview with the Fields medalist Sergei Novikov, 19.12.2016, http://www.kommersant.ru/doc/3169063 (in Russian). 